\title{
Optimal Control of a Dengue-Dengvaxia Model: Comparison Between Vaccination and Vector Control
}

https://doi.org/10.1515/cmb-2020-0124

Received June 17, 2021; accepted October 19, 2021

Abstract: Dengue is the most common mosquito-borne viral infection transmitted disease. It is due to the four types of viruses (DENV-1, DENV-2, DENV-3, DENV-4), which transmit through the bite of infected Aedes aegypti and Aedes albopictus female mosquitoes during the daytime. The first globally commercialized vaccine is Dengvaxia, also known as the CYD-TDV vaccine, manufactured by Sanofi Pasteur. This paper presents a Ross-type epidemic model to describe the vaccine interaction between humans and mosquitoes using an entomological mosquito growth population and constant human population. After establishing the basic reproduction number $\mathcal{R}_{0}$, we present three control strategies: vaccination, vector control, and the combination of vaccination and vector control. We use Pontryagin's minimum principle to characterize optimal control and apply numerical simulations to determine which strategies best suit each compartment. Results show that vector control requires shorter time applications in minimizing mosquito populations. Whereas vaccinating the primary susceptible human population requires a shorter time compared to the secondary susceptible human.

Keywords: Dengvaxia; Vaccination; $\mathcal{R}_{0}$; Optimal Control; Pontryagin maximum principle

MSC: 34D23; 92D30; 92-10; 49J15

\section{Introduction}

Dengue is a mosquito-borne viral infection found in tropical and subtropical regions around the world. It is the most common viral infection transmitted disease. In a study by [4] an estimated 96 million apparent dengue infections globally in 2010 predominantly, 70\% in Asia. In the Philippines alone, 271,480 dengue cases were reported nationwide from January 1 to August 31, 2019. [8] It occurs nationally in the Philippines, including urban and peri-urban areas, with peak transmission during the rainy season.

Dengue is a viral infection caused by four types of viruses (DENV-1, DENV-2, DENV-3, DENV-4), which transmit through the bite of infected Aedes aegypti and Aedes albopictus female mosquitoes during the daytime. In most cases, dengue is a self-limiting illness. However, it may require hospital admission, where supportive care can modify the course of the illness. Infection from one type grants life-long immunity to that virus strain and also appears to grant partial protection against the other types temporarily. That is, when infected for a second time with a different type of virus, a more severe form of the disease will occur, known as Dengue Hemorrhagic Fever (DHF). In the Philippines alone, 1,107 deaths are reported from January 1 to August 31, 2019, due to dengue fever [8].

\footnotetext{
${ }^{\star}$ Corresponding Author: Cheryl Q. Mentuda: Laboratoire Amiénois de Mathématique Fondamentale et Appliquée, CNRS UMR 7352, Université de Picardie Jules Verne, 80069 Amiens, France Department of Mathematics, Caraga State University, Butuan City, Philippines, E-mail: cheryl.mentuda@u-picardie.fr
} 
Efforts to develop a vaccine against dengue have been ongoing for decades. The first such vaccine to be used commercially is CYD-TDV, marketed as Dengvaxia by Sanofi Pasteur. Dengvaxia was licensed in December 2015 and approved in 11 countries. One such country is the Philippines. On December 1, 2015, the commercial sale of Dengvaxia was approved, making the Philippines the first Asian country to commercialize Dengvaxia [12]. In effect, in April 2016, the Department of Health (DOH) launched the dengue vaccination campaign in the Philippine regions of Central Luzon, Calabarzon, and Metro Manila, involving more than 800,000 school children who received at least one dose of the vaccine. Dengvaxia is a live vaccine of serotypes $1,2,3$, and 4 [18]. It is a live attenuated chimeric product made using recombinant DNA technology by replacing the PrM (pre-membrane) and E (envelope) structural genes of yellow fever attenuated 17D strain vaccine with those from the four dengue serotypes. It should be administered in three doses of $0.5 \mathrm{~mL}$ subcutaneous (SC) six months apart. Sanofi Pasteur recommended that the vaccine only be used in people between the age of 9 to 45 and people already infected by one type of virus [2]. It is because outcomes may be worse in those who have not yet been infected.

Mathematical modeling has been used to test and determine the effectiveness of different intervention strategies in controlling dengue. SEIRS compartmental models accounting for Susceptible, Exposed, Infected, and Removed for the human population, and susceptible and infected for mosquito population were widely promoted. [14] studied a system of differential equations that models the population dynamics of SEIR vector transmission of dengue fever. While [7] proposed a model with two different viruses acting at separated intervals of time. They study the dynamics of dengue fever while concentrating on its progression to the hemorrhagic form to understand the epidemic phenomenon and suggest strategies for the control of the disease. Furthermore, [11] derived and analyzed the model taking into account the severe DHF compartment in the transmission model. They find a control measure to reduce the DHF patients in the population or keep the number of patients at an acceptable level. Also, [1] surveyed a model framework for dengue fever epidemiology. They discuss the role of several subsequent infections versus a detailed number of dengue serotypes included in the model framework and the human immunological aspects associated with disease severity, identifying the implications for model dynamics and their impact on vaccine implementation.

To determine the optimal control in minimizing the spread of dengue fever has also been studied. [17] described the dynamics of dengue disease in the compartment model, taking into account chemical controls and mechanical control applied on the mosquitoes. [13] presented a control mechanism based on a dengue model with vertical transmission considering the two policies, namely vaccination, and insecticide administration. [6] evaluated a control strategy, which aims to eliminate the Aedes aegypti mosquito, as well as proposals for the vaccination campaign. Their results show that eradicating dengue fever is done using an immunizing vaccine since control measures against its vector are not enough to stop the disease from spreading. Moreover, [9] designed a new mathematical model to assess the impact of the newly- released Dengvaxia vaccine on the transmission dynamics of two co-circulating dengue strains.

This paper is the first study to take into account the proposal of Sanofi Pasteur. Herein we introduce a new mathematical model of Dengvaxia. As recommended by the World Health Organization [16], vaccination should be given to individuals who have been already infected by one strain virus. In this paper, we split the susceptible human compartment into primary or secondary susceptible humans, that is, individuals who have not been infected and individuals who have been infected by one or more strains of the dengue virus. Then we present a Ross-type epidemic model describing the interaction between humans and the mosquito population. It is the first dengue model that uses the entomological growth function for the mosquito population. We first compare three control strategies concerning the reproduction number: vaccination, vector control, and the combination of the two. Moreover, the optimal control of the three methods is computed to determine the best strategy for the population. 


\section{The model and its analysis}

\subsection{Description of model with vaccination}

We assumed that dengue viruses are virulent and that no other microorganism attacks the body. The model is based on the Ross-type model. Let $H$ be the human population subdivided into primary susceptible $S_{h}$, secondary susceptible $\widetilde{S}_{h}$, infected $I_{h}$ and removed $R_{h}$. Primary susceptible humans are individuals who have not yet been infected by dengue. In contrast, secondary susceptible humans are individuals who previously had dengue infections. Let $M$ be the population of females mosquitoes split into two groups of susceptible $S_{m}$ and infectious $I_{m}$ mosquitoes. Figure 1 describes the flow of dengue disease.

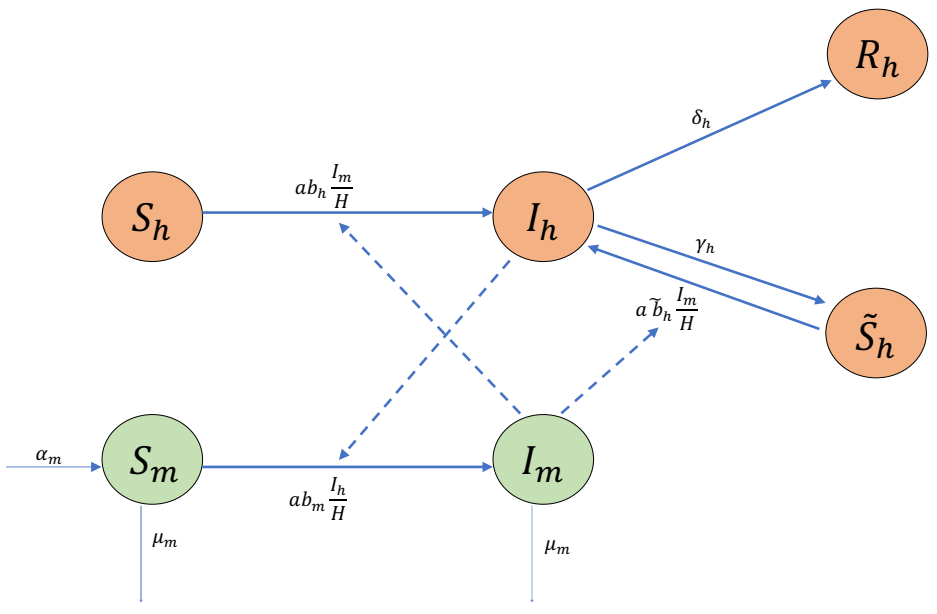

Figure 1: Compartmental representation of the dynamics of dengue virus in the human and mosquito population.

Since human have a meager mortality rate compared to mosquitoes, we neglect the natural death of the humans. The following system of ordinary equations governed the dynamics of humans:

$$
\begin{aligned}
S_{h}^{\prime}(t) & =-\frac{a b_{h} I_{m}(t)}{H(t)} S_{h}(t) \\
I_{h}^{\prime}(t) & =\frac{a I_{m}(t)}{H(t)}\left(b_{h} S_{h}(t)+\widetilde{b}_{h} \widetilde{S}_{h}(t)\right)-\gamma_{h} I_{h}(t)-\delta_{h} I_{h}(t) \\
\widetilde{S}_{h}^{\prime}(t) & =\gamma_{h} I_{h}(t)-\frac{a \widetilde{b}_{h} I_{m}(t)}{H(t)} \widetilde{S}_{h}(t) \\
R_{h}^{\prime}(t) & =\delta_{h} I_{h}(t)
\end{aligned}
$$

while the dynamics of mosquitoes follows:

$$
\begin{aligned}
& S_{m}^{\prime}(t)=-\frac{a b_{m} I_{h}(t)}{H(t)} S_{m}(t)-\mu_{m} S_{m}(t)+g(M(t)) \\
& I_{m}^{\prime}(t)=\frac{a b_{m} I_{h}(t)}{H(t)} S_{m}(t)-\mu_{m} I_{m}(t) .
\end{aligned}
$$

Note that the total population of human is $H=S_{h}+I_{h}+\widetilde{S}_{h}+R_{h}$ and the total population of mosquito is $M=S_{m}+I_{m}$. In this work, we consider constant human population, which is $H^{\prime}(t)=0$, and an entomological growth function for mosquito population, which is $M^{\prime}(t)=g(M(t))-\mu_{m} M(t)=\alpha_{m} M(t) e^{-\beta_{m} M(t)}-\mu_{m} M(t)$ as in [5], where $\mu_{m}$ is the death rate and $\alpha_{m}$ is the natural birth rate of the mosquitoes. 
The parameters $a b_{h} I_{m} \frac{S_{h}}{H}$ represent the infection rate of the proportion of primary susceptible individuals to be infected with the dengue virus. Wherein $b_{h}$ is the probability of transmission of the virus from an infected mosquito to a primary susceptible human, and $a$ represents a mosquito's average bites. Moreover, $a \widetilde{b}_{h} I_{m} \frac{\widetilde{S}_{h}}{H}$ is the infection rate of the proportion of susceptible individuals who had been previously infected with dengue to become infectious again with different serotypes, $\widetilde{b}_{h}$ is the probability of transmission of the virus from an infected mosquito to secondary susceptible human. Furthermore, the rate of susceptible secondary people recovering from infection from one serotype is represented by $\gamma_{h} I_{h}(t)$.

For the mosquito compartment, $g(M(t))$ is the growth of the total mosquito population, and $a b_{m} S_{m} \frac{I_{h}}{H}$ is the infection rate of susceptible mosquitoes to be infectious once it bites a ratio of the infected human population. The parameter $b_{m}$ is the transmission probability from an infected human to a susceptible mosquito.

\subsection{Well-posedness and positivity of solution}

To simplify the reading, the system of ordinary differential equations is rewritten as $U^{\prime}(t)=F(t, u(t))$ with

$$
U(t)=\left(u_{1}=S_{h}, u_{2}=I_{h}, u_{3}=\widetilde{S}_{h}, u_{4}=R_{h}, u_{5}=S_{m}, u_{6}=I_{m}\right)^{t}
$$

and

$$
\begin{aligned}
F(U(t), t)= & \left(-\frac{a b_{h} u_{6} u_{1}}{H}, \frac{a u_{6}\left(b_{h} u_{1}+\widetilde{b}_{h} u_{3}\right)}{H}-\gamma_{h} u_{2}-\delta_{h} u_{2}, \gamma_{h} u_{2}-\frac{a \widetilde{b}_{h} u_{6} u_{3}}{H}, \delta_{h} u_{2},\right. \\
& \left.-\frac{a b_{m} u_{2} u_{5}}{H}-\mu_{m} u_{5}+g(M), \frac{a b_{m} u_{2} u_{5}}{H}-\mu_{m} u_{6}\right)^{t} .
\end{aligned}
$$

Theorem 2.1. The domain $\Omega$ defined by

$$
\Omega=\left\{U \in \mathbb{R}_{+}^{6}: 0 \leq u_{1}+u_{2}+u_{3}+u_{4}=H_{0}, 0 \leq u_{5}+u_{6} \leq \max \left(\frac{\alpha_{m}}{\beta_{m} \mu_{m}}, M_{0}\right)\right\}
$$

is positively invariant. In particular, for an initial datum $U(0)$ in $\Omega$, there exists a unique global in time solution $U$ in $\mathcal{C}\left(\mathbb{R}_{+}, \Omega\right)$.

Proof. Consider the initial value problem

$$
U^{\prime}(t)=F(t, U(t)) \text { where } \quad U(0)=U_{0} .
$$

The right-hand-side $F$ satisfies the local Lipschitz condition. Therefore, the Cauchy-Lipschitz theorem ensure the local well-posedness. Then,

$$
\begin{aligned}
& f_{1}\left(0, u_{2}, u_{3}, u_{4}, u_{5}, u_{6}\right)=0, \forall u_{2}, \cdots, u_{6} \in \Omega \\
& f_{2}\left(u_{1}, 0, u_{3}, u_{4}, u_{5}, u_{6}\right)=\frac{a u_{6}\left(b_{h} u_{1}+\widetilde{b}_{h} u_{3}\right)}{H} \geq 0, \forall u_{1}, u_{3}, \cdots, u_{6} \in \Omega \\
& f_{3}\left(u_{1}, u_{2}, 0, u_{4}, u_{5}, u_{6}\right)=\gamma_{h} u_{2}, \forall u_{1}, u_{2}, u_{4}, \cdots, u_{6} \in \Omega \\
& f_{4}\left(u_{1}, u_{2}, u_{3}, 0, u_{5}, u_{6}\right)=\delta_{h} u_{2}, \forall u_{1}, u_{2}, u_{3}, u_{5}, u_{6} \in \Omega \\
& f_{5}\left(u_{1}, u_{2}, u_{3}, u_{4}, 0, u_{6}\right)=\alpha_{m} u_{6} e^{-\beta_{m} u_{6}}, \forall u_{1}, \cdots, u_{4}, u_{6} \in \Omega \\
& f_{6}\left(u_{1}, u_{2}, u_{3}, u_{4}, u_{5}, 0\right)=\frac{a b_{m} u_{2} u_{5}}{H}, \forall u_{1}, \cdots, u_{5} \in \Omega
\end{aligned}
$$

On one hand, note that $H^{\prime}=u_{1}^{\prime}+u_{2}^{\prime}+u_{3}^{\prime}+u_{4}^{\prime}=0$, and $u_{1}+u_{2}+u_{3}+u_{4}=H_{0}$. On the other hand

$$
u_{5}^{\prime}+u_{6}^{\prime}=\alpha_{m}\left(u_{5}+u_{6}\right) e^{-\beta_{m}\left(u_{5}+u_{6}\right)}-\mu_{m}\left(u_{5}+u_{6}\right) \text {, }
$$


or

$$
M^{\prime}=\alpha_{m} M e^{-\beta_{m} M}-\mu_{m} M
$$

If $\mu_{m} \geq \alpha_{m}$, using $e^{-\beta_{m} M} \leq 1$, then

$$
M^{\prime} \leq\left(\alpha_{m}-\mu_{m}\right) M .
$$

From Grönwall's Lemma, with $\alpha_{m}-\mu_{m} \leq 0$

$$
M(t) \leq e^{\left(\alpha_{m}-\mu_{m}\right) t} M_{0} \leq M_{0} .
$$

If $\alpha_{m}>\mu_{m}$, then

$$
\begin{aligned}
\alpha_{m} M e^{-\beta_{m} M} & =\frac{\alpha_{m} M}{e^{\beta_{m} M}}=\frac{\alpha_{m} M}{\sum_{n \geq 0} \frac{\left(\beta_{m} M\right)^{n}}{n !}} \\
& =\frac{\alpha_{m} M}{1+\beta_{m} M+\frac{\beta_{m}^{2} M^{2}}{2}+\frac{\beta_{m}^{3} M^{3}}{6}+\cdots} \\
& \leq \frac{\alpha_{m}}{\beta_{m}}
\end{aligned}
$$

Then $M^{\prime} \leq \frac{\alpha_{m}}{\beta_{m}}-\mu_{m} M$ and by Gronwall's lemma $M(t) \leq e^{-\mu_{m} t}\left(M_{0}-\frac{\alpha_{m}}{\beta_{m} \mu_{m}}\right)+\frac{\alpha_{m}}{\beta_{m} \mu_{m}} \leq M_{0}$.

\subsection{Stability of equilibrium and Basic Reproduction Number $\mathcal{R}_{0}$}

In this section, we determine the possible equilibrium points of the model and study their behavior. Let $\left(u_{1}^{\star}, u_{2}^{\star}, u_{3}^{\star}, u_{4}^{\star}, u_{5}^{\star}, u_{6}^{\star}\right) \in \Omega$. Solving the system

$$
\begin{aligned}
&-\frac{a b_{h} u_{6}^{\star} u_{1}^{\star}}{H_{0}}=0 \\
& \frac{a u_{6}^{\star}\left(b_{h} u_{1}^{\star}+\widetilde{b}_{h} u_{3}^{\star}\right)}{H_{0}}-\gamma_{h} u_{2}^{\star}-\delta_{h} u_{2}^{\star}=0 \\
& \gamma_{h} u_{2}^{\star}-\frac{a \widetilde{b}_{h} u_{3}^{\star} u_{6}^{\star}}{H_{0}}=0 \\
& \delta_{h} u_{2}^{\star}=0 \\
&-\frac{a b_{m} u_{2}^{\star} u_{5}^{\star}}{H_{0}}-\mu_{m} u_{5}^{\star}+\alpha_{m} M e^{-\beta_{m} M}=0 \\
& \frac{a b_{m} u_{2}^{\star} u_{5}^{\star}-\mu_{m} u_{6}^{\star}}{H_{0}}=0
\end{aligned}
$$

we get two disease free equilibrium $E_{1}=\left(u_{1}^{\star}, 0, u_{3}^{\star}, u_{4}^{\star}, 0,0\right)$ and $E_{2}=\left(u_{1}^{\star}, 0, u_{3}^{\star}, u_{4}^{\star}, \frac{1}{\beta_{m}} \ln \left(\frac{\alpha_{m}}{\mu_{m}}\right), 0\right)$. Note that the equilibrium $E_{2}$ is biologically relevant only when $\alpha_{m}>\mu_{m}$.

Since the infected individuals are in $u_{2}$ and $u_{6}$, then we can rewrite the system of the equations as

$$
\mathcal{F}=\left(\begin{array}{c}
\frac{a u_{6}\left(b_{h} u_{1}+\widetilde{b}_{h} u_{3}\right)}{H_{0}} \\
\frac{a b_{m} u_{2} u_{5}}{H_{0}}
\end{array}\right) \quad \mathcal{V}=\left(\begin{array}{c}
\left(\gamma_{h}+\delta_{h}\right) u_{2} \\
\mu_{m} u_{6}
\end{array}\right)
$$

where $\mathcal{F}$ is the rate of appearance of new infections in each compartment and $\mathcal{V}$ is the rate of other transitions between all compartments. Thus,

$$
F=\left(\begin{array}{cc}
0 & \frac{a\left(b_{h} u_{1}+\widetilde{b}_{h} u_{3}\right)}{H_{0}} \\
\frac{a b_{m} u_{5}}{H_{0}} & 0
\end{array}\right)
$$


and $V=\left(\begin{array}{cc}\gamma_{h}+\delta_{h} & 0 \\ 0 & \mu_{m}\end{array}\right)$ where $V^{-1}=\left(\begin{array}{cc}\frac{1}{\gamma_{h}+\delta_{h}} & 0 \\ 0 & \frac{1}{\mu_{m}}\end{array}\right)$. Therefore, the next generation matrix is

$$
F V^{-1}=\left(\begin{array}{cc}
0 & \frac{a\left(b_{h} u_{1}+\widetilde{b}_{h} u_{3}\right)}{\mu_{m} H_{0}} \\
\frac{a b_{m} u_{5}}{\left(\gamma_{h}+\delta_{h}\right) H_{0}} & 0
\end{array}\right) \text {. }
$$

It follows that by [15] the basic reproduction number, denoted by $\rho\left(F V^{-1}\right)$, where $\rho$ is the spectral radius, is given by

$$
\rho\left(F V^{-1}\right)=\sqrt{\frac{a^{2} b_{m} u_{5}\left(b_{h} u_{1}+\widetilde{b}_{h} u_{3}\right)}{\mu_{m} H_{0}^{2}\left(\gamma_{h}+\delta_{h}\right)}} .
$$

Now using this eigenvalue, we determine the local stability of the equilibrium points $E_{1}$ and $E_{2}$.

\section{Proposition 2.2.}

1. The disease free equilibrium $E_{1}=\left(u_{1}^{\star}, 0, u_{3}^{\star}, u_{4}^{\star}, 0,0\right)$ is locally asymptotically stable.

2. If $\alpha_{m}>\mu_{m}$ and $\mathcal{R}_{0}<1$ where $\mathcal{R}_{0}=\sqrt{\frac{a^{2} b_{m} \ln \left(\frac{\alpha_{m}}{\mu_{m}}\right)\left(b_{h} u_{1}^{*}+\widetilde{b}_{h} u_{3}^{*}\right)}{H_{0}^{2} \mu_{m} \beta_{m}\left(\gamma_{h}+\delta_{h}\right)}}$, then the disease free equilibrium point $E_{2}=\left(u_{1}^{\star}, 0, u_{3}^{\star}, u_{4}^{\star}, \frac{1}{\beta_{m}} \ln \left(\frac{\alpha_{m}}{\mu_{m}}\right), 0\right)$ is locally asymptotically stable.

Probf. From the above eigenvalues,

$$
\rho\left(F V^{-1}\right)=\sqrt{\frac{a^{2} b_{m}(0)\left(\widetilde{b}_{h} u_{3}^{\star}+b_{h} u_{1}^{\star}\right)}{H_{0}^{2} \mu_{m}\left(\gamma_{h}+\delta_{h}\right)}}=0
$$

Therefore, the system of equation is local asymptotically stable at $E_{1}$.

2. Similarly,

$$
\mathcal{R}_{0}=\sqrt{\frac{a^{2} b_{m} u_{5}^{\star}\left(b_{h} u_{1}^{\star}+\widetilde{b}_{h} u_{3}^{\star}\right)}{\mu_{m} H_{0}^{2}\left(\gamma_{h}+\delta_{h}\right)}}=\sqrt{\frac{a^{2} b_{m} \ln \left(\frac{\alpha_{m}}{\mu_{m}}\right)\left(\widetilde{b}_{h} u_{3}^{\star}+b_{h} u_{1}^{\star}\right)}{\mu_{m} H_{0}^{2} \beta_{m}\left(\gamma_{h}+\delta_{h}\right)}} .
$$

The basic reproduction number $\mathcal{R}_{0}$ has a biological meaning when $\alpha_{m}>\mu_{m}$. It means that the average number of new infected humans is proportional to the proportion $\frac{u_{5}^{*}}{H_{0}}$ of susceptible mosquitoes among the human population. The terms $\frac{u_{1}^{*}}{H_{0}}$, and $\frac{u_{3}^{*}}{H_{0}}$ proportion of primary and secondary susceptible humans respectively. The terms $\frac{a b_{h}}{\mu_{m}\left(\gamma_{h}+\delta_{h}\right)}$, and $\frac{a \widetilde{b}_{h}}{\mu_{m}\left(\gamma_{h}+\delta_{h}\right)}$ represent the transmission rate due by biting during the infection period $1 /\left(\gamma_{h}+\delta_{h}\right)$ and mosquitoes life expectancy $1 / \mu_{m}$.

Remark 2.3. Local stability can be proved using the Jacobian matrix defined as

$$
J=\left(\begin{array}{cccccc}
\frac{-a b_{h} u_{6}}{H_{0}} & 0 & 0 & 0 & 0 & \frac{-a b_{h} u_{1}}{H_{0}} \\
\frac{a b_{h} u_{6}}{H_{0}} & -\gamma_{h}-\delta_{h} & \frac{a \widetilde{b}_{h} u_{6}}{H_{0}} & 0 & 0 & \frac{a b_{h} u_{1}+a \widetilde{b}_{h} u_{3}}{H_{0}} \\
0 & \gamma_{h} & \frac{-a \bar{b}_{h} u_{6}}{H_{0}} & 0 & 0 & \frac{-a b_{h} u_{3}}{H_{0}} \\
0 & \delta_{h} & 0 & 0 & 0 & 0 \\
0 & \frac{-a b_{m} u_{5}}{H_{0}} & 0 & 0 & \frac{-a b_{m} u_{2}}{H_{0}}-\mu_{m}+\alpha_{m} e^{-\beta_{m} M}\left(1-\beta_{m} M\right) & \alpha_{m} e^{-\beta_{m} M}\left(1-\beta_{m} M\right) \\
0 & \frac{a b_{m} u_{5}}{H_{0}} & 0 & 0 & \frac{a b_{m} u_{2}}{H_{0}} & -\mu_{m}
\end{array}\right)
$$

Concerning the disease free equilibrium $E_{1}=\left(u_{1}^{\star}, 0, u_{3}^{\star}, u_{4}^{\star}, 0,0\right)$, we get as eigenvalues

$$
\begin{aligned}
& \lambda_{1}=0 \text { with multiplicity } 3 \\
& \lambda_{2}=-\gamma_{h}-\delta_{h} \\
& \lambda_{3}=-\mu_{m} \\
& \lambda_{4}=-\mu_{m}+\alpha_{m}
\end{aligned}
$$


If $\alpha_{m}-\mu_{m} \leq 0$, all the eigenvalues are negative. Implying further that the system of equation is asymptotically stable at the equilibrium $E_{1}$.

For the second equilibrium $E_{2}=\left(u_{1}^{\star}, 0, u_{3}^{\star}, u_{4}^{\star}, \frac{1}{\beta_{m}} \ln \left(\frac{\alpha_{m}}{\mu_{m}}\right), 0\right)$, the eigenvalues are

$$
\begin{aligned}
& \lambda=0 \quad \text { with multiplicity } 3 \\
& \lambda=-\mu_{m} \ln \left(\frac{\alpha_{m}}{\mu_{m}}\right) \\
& \lambda=-\frac{\gamma_{h}+\delta_{h}+\mu_{m}}{2} \pm \frac{\sqrt{\left(\gamma_{h}+\delta_{h}+\mu_{m}\right)^{2}-4\left(\mu_{m}\left(\gamma_{h}+\delta_{h}\right)-\frac{a b_{m} \ln \left(\frac{\alpha_{m}}{\mu_{m}}\right)\left(a b_{h} u_{1}^{*}+a \widetilde{b}_{h} u_{3}^{*}\right)}{H_{0}{ }^{2} \beta_{m}}\right)}}{2}
\end{aligned}
$$

which are negative if $\alpha_{m}>\mu_{m}$ and $\mathcal{R}_{0}>1$.

\section{Theorem 2.4.}

1. If $\alpha_{m}<\mu_{m}$, then $E_{1}$ is globally asymptotically stable.

2. If $\alpha_{m}>\mu_{m}$ and $\mathcal{R}_{0}>1$, then $E_{2}$ is globally asymptotically stable.

Proof. From equation (4), we can deduce that $u_{4}$ is increasing. Since $u_{4}$ is bounded by $H_{0}, u_{4}$ has a limit $u_{4}^{\star}$ as $t \rightarrow+\infty$. Thus, integrating equation (4) gives

$$
u_{4}(t)-u_{4}(0)=\delta_{h} \int_{0}^{t} u_{2}(s) d s .
$$

Thus

$$
u_{4}^{\star}-u_{4}(0)=\delta_{h} \int_{0}^{\infty} u_{2}(s) d s
$$

which is finite. Implying further that $u_{2}(s) \rightarrow 0$ as $s \rightarrow+\infty$. Now, adding equation (5) and (6) gives us

$$
u_{5}^{\prime}+u_{6}^{\prime}=M^{\prime}=g(M)=\alpha_{m} M e^{-\beta_{m} M}-\mu_{m} M .
$$

As in Theorem 2.1, if $\alpha_{m}<\mu_{m}$, then $M(t) \rightarrow 0$ as $t \rightarrow+\infty$. Thus, by positivity of the solution $u_{5}$ and $u_{6}$ goes to 0 as $t \rightarrow+\infty$. From equation (1) $u_{1}^{\prime}=-\frac{a b_{h} u_{6} u_{1}}{H_{0}} \leq 0$, the function $u_{1}$ is decreasing non-negative function, bounded by $H_{0}$. Thus, as $t \rightarrow+\infty, u_{1} \rightarrow u_{1}^{\star}$. The solution of equation (3) $u_{3}^{\prime}+\frac{a \widetilde{b}_{h} u_{6} u_{3}}{H_{0}}=\gamma_{h} u_{2}$, can be written as

$$
u_{3}(t)=E(t) u_{3}(0)+\int_{0}^{t} E(t-s) \gamma_{h} u_{2}(s) d s
$$

with $E(t)=e^{-\frac{a \tilde{b}_{h}}{H_{0}} \int_{0}^{t} u_{6}(s) d s}$. Since, for all $t \geq 0,0 \leq u_{2}(t), u_{3}(t) \leq H_{0}, 0 \leq u_{6}(t) \leq M_{0}$, then $u_{2} \rightarrow 0, u_{3} \rightarrow u_{3}^{\star}$ when $t \rightarrow+\infty$.

When $\alpha_{m} \geq \mu_{m}$, let us denote $M^{\star}=\frac{1}{\beta_{m}} \ln \left(\frac{\alpha_{m}}{\mu_{m}}\right)$. Since $M^{\prime}(t)=\left(\alpha_{m} e^{-\beta M}-\mu_{m}\right) M$, if $M(t)<M^{\star}$, then $M$ is increasing and bounded by above, while if $M(t)>M^{\star}$, then $M$ is decreasing and bounded by below. In particular, $M(t)$ has a limit when $t \rightarrow+\infty$. Using now the local asymptotic stability with $\mathcal{R}_{0}<1$, this limit is equal to $M^{\star}$.

\section{Comparison of control strategies}

In this section, we describe three control strategies in preventing or reducing dengue transmission. 


\subsection{Vaccination}

To account for vaccine in the model, let us consider the following mathematical model:

$$
\begin{aligned}
& u_{1}^{\prime}(t)=-\frac{a b_{h} u_{6}(t) u_{1}(t)}{H_{0}}-v_{1} u_{1}(t) \\
& u_{2}^{\prime}(t)=\frac{a u_{6}(t)\left(b_{h} u_{1}(t)+\widetilde{b}_{h} u_{3}(t)\right)}{H_{0}}-\gamma_{h} u_{2}(t)-\delta_{h} u_{2}(t) \\
& u_{3}^{\prime}(t)=\gamma_{h} u_{2}(t)-\frac{a \widetilde{b}_{h} u_{3}(t) u_{6}(t)}{H_{0}}-v_{3} u_{3}(t) \\
& u_{4}^{\prime}(t)=\delta_{h} u_{2}(t) \\
& u_{5}^{\prime}(t)=-\frac{a b_{m} u_{2}(t) u_{5}(t)}{H_{0}}-\mu_{m} u_{5}(t)+\alpha_{m} M e^{-\beta_{m} M} \\
& u_{6}^{\prime}(t)=\frac{a b_{m} u_{2}(t) u_{5}(t)}{H_{0}}-\mu_{m} u_{6}(t) .
\end{aligned}
$$

We distinguish the vaccination given to the primary susceptible human population $\left(v_{1} u_{1}\right)$, and the vaccination given to the secondary susceptible human $\left(v_{3} u_{3}\right)$. The total immunity is given by $T_{h}^{\prime}(t)=v_{1} u_{1}(t)+v_{3} u_{3}(t)$.

Note that there exists a unique global in time solution $\left(u_{1}, u_{2}, u_{3}, u_{4}, u_{5}, u_{6}\right)$ in $\mathcal{C}\left(\mathbb{R}_{+}, \mathbb{R}_{+}\right)^{6}$. And the system of equations (8) admits two equilibria $E_{1, v a c}=\left(0,0,0, u_{4}^{\star}, 0,0\right)$ and $E_{2, v a c}=$ $\left(0,0,0, u_{4}^{\star}, \frac{1}{\beta_{m}} \ln \left(\frac{\alpha_{m}}{\mu_{m}}\right), 0\right)$.

The next generation matrix does not change, and the basic reproduction number is $\mathcal{R}_{v a c}=0$. Therefore, we have the following theorem.

Theorem 3.1. 1. If $\alpha_{m}<\mu_{m}$, then $E_{1, v a c}$ is globally asymptotically stable.

2. If $\alpha_{m}>\mu_{m}$, then $E_{2, v a c}$ is globally asymptotically stable.

Proof. The proof is similar to the proof of Theorem 2.4 by replacing $\frac{a b_{h} u_{6}}{H_{0}}$ by $\left(\frac{a b_{h} u_{6}}{H_{0}}+v_{1}\right)$ and $\frac{a \widetilde{b}_{h} u_{6}}{H_{0}}$ by $\left(\frac{a \widetilde{b}_{h} u_{6}}{H_{0}}+v_{3}\right)$.

\subsection{Vector Control}

Vector control consists in limiting the vectors that transmit disease. The most frequent type of vector control uses various strategies such as habitat and environmental control, reducing vector contact, chemical control, and biological control. To include vector control, let us consider a mathematical model below:

$$
\begin{aligned}
& u_{1}^{\prime}(t)=-\frac{a b_{h} u_{6}(t) u_{1}(t)}{H_{0}} \\
& u_{2}^{\prime}(t)=\frac{a u_{6}(t)\left(b_{h} u_{1}(t)+\widetilde{b}_{h} u_{3}(t)\right)}{H_{0}}-\gamma_{h} u_{2}(t)-\delta_{h} u_{2}(t) \\
& u_{3}^{\prime}(t)=\gamma_{h} u_{2}(t)-\frac{a \widetilde{b}_{h} u_{3}(t) u_{6}(t)}{H_{0}} \\
& u_{4}^{\prime}(t)=\delta_{h} u_{2}(t) \\
& u_{5}^{\prime}(t)=-\frac{a b_{m} u_{2}(t) u_{5}(t)}{H_{0}}-\mu_{m} u_{5}(t)+\alpha_{m} M e^{-\beta_{m} M}-v_{m} u_{5}(t) \\
& u_{6}^{\prime}(t)=\frac{a b_{m} u_{2}(t) u_{5}(t)}{H_{0}}-\mu_{m} u_{6}(t)-v_{m} u_{6}(t)
\end{aligned}
$$

where $v_{m} u_{5}$ is the vector control given to the susceptible mosquito population, and $v_{m} u_{6}$ is the vector control to infectious mosquito. The total controlled mosquito is given by $T_{m}^{\prime}(t)=v_{m}\left(u_{5}(t)+u_{6}(t)\right)$. 
Again, there exists a unique global in time solution $\left(u_{1}, u_{2}, u_{3}, u_{4}, u_{5}, u_{6}\right)$ in $\mathcal{C}\left(\mathbb{R}_{+}, \mathbb{R}_{+}\right)^{6}$, and the system admits two equilibria $E_{1, v e c}=\left(u_{1}^{\star}, 0, u_{3}^{\star}, u_{4}^{\star}, 0,0\right)$ and $E_{2, v e c}=\left(u_{1}^{\star}, 0, u_{3}^{\star}, u_{4}^{\star}, \frac{1}{\beta_{m}} \ln \left(\frac{\alpha_{m}}{v_{m}+\mu_{m}}\right), 0\right)$.

Here the basic reproduction number is

$$
\mathcal{R}_{\text {vec }}=\sqrt{\frac{a^{2} b_{m} \frac{1}{\beta_{m}} \ln \left(\frac{\alpha_{m}}{\mu_{m}+v_{m}}\right)\left(\widetilde{b}_{h} u_{3}^{\star}+b_{h} u_{1}^{\star}\right)}{H_{0}^{2}\left(\mu_{m}+v_{m}\right)\left(\gamma_{h}+\delta_{h}\right)}} .
$$

Theorem 3.2. 1. If $\alpha_{m}<\mu_{m}+v_{m}$, then $E_{1, v e c}$ is globally asymptotically stable.

2. If $\alpha_{m}>\mu_{m}+v_{m}$ and $\mathcal{R}_{v e c}>1$, then $E_{2, v e c}$ is globally asymptotically stable.

\subsection{Vaccination and Vector Control}

When the dengue vaccination and the vector control method are combined, we have:

$$
\begin{aligned}
& u_{1}^{\prime}(t)=-\frac{a b_{h} u_{6}(t) u_{1}(t)}{H_{0}}-v_{1} u_{1}(t) \\
& u_{2}^{\prime}(t)=\frac{a u_{6}(t)\left(b_{h} u_{1}(t)+\widetilde{b}_{h} u_{3}(t)\right)}{H_{0}}-\gamma_{h} u_{2}(t)-\delta_{h} u_{2}(t) \\
& u_{3}^{\prime}(t)=\gamma_{h} u_{2}(t)-\frac{a \widetilde{b}_{h} u_{3}(t) u_{6}(t)}{H_{0}}-v_{3} u_{3}(t) \\
& u_{4}^{\prime}(t)=\delta_{h} u_{2}(t) \\
& u_{5}^{\prime}(t)=-\frac{a b_{m} u_{2}(t) u_{5}(t)}{H_{0}}-\mu_{m} u_{5}(t)+\alpha_{m} M e^{-\beta_{m} M}-v_{m} u_{5}(t) \\
& u_{6}^{\prime}(t)=\frac{a b_{m} u_{2}(t) u_{5}(t)}{H_{0}}-\mu_{m} u_{6}(t)-v_{m} u_{6}(t)
\end{aligned}
$$

where total human immunity is given by $T_{h}^{\prime}(t)=v_{1} u_{1}(t)+v_{3} u_{3}(t)$ and total vector control is given by $T_{m}^{\prime}(t)=$ $v_{m} u_{5}(t)+v_{m} u_{6}(t)$.

There exists a unique global in time solution $\left(u_{1}, u_{2}, u_{3}, u_{4}, u_{5}, u_{6}\right)$ in $\mathcal{C}\left(\mathbb{R}_{+}, \mathbb{R}_{+}\right)^{6}$, and the system admits two equilibria $E_{1, \text { both }}=\left(0,0,0, u_{4}^{\star}, 0,0\right)$ and $E_{2, \text { both }}=\left(0,0,0, u_{4}^{\star}, \frac{1}{\beta_{m}} \ln \left(\frac{\alpha_{m}}{\mu_{m}+v_{m}}\right), 0\right)$.

Theorem 3.3. 1. If $\alpha_{m}<\mu_{m}+v_{m}$, then $E_{1, b o t h}$ is globally asymptotically stable.

2. If $\alpha_{m}>\mu_{m}+v_{m}$, then $E_{2, \text { both }}$ is globally asymptotically stable.

\section{Optimal Control Problem}

\subsection{Minimizing Infected Humans by Optimal Vaccination and Vector Control}

Assume that both control inputs are piecewise continuous functions that take its values in a positively bounded set $W=\left[0, w_{H}\right]^{2} \times\left[0, w_{M}\right]^{2}$. Thus we consider the objective function

$$
\mathcal{\partial}\left(w_{1}, w_{3}, w_{m}\right)=\int_{0}^{T} u_{2}(t)+\frac{1}{2}\left(A_{1} w_{1}^{2}(t)+A_{3} w_{3}^{2}(t)+A_{m} w_{m}^{2}(t)\right) d t
$$


subject to

$$
\begin{aligned}
& u_{1}^{\prime}(t)=-\frac{a b_{h} u_{6}(t) u_{1}(t)}{H_{0}}-w_{1}(t) u_{1}(t) \\
& u_{2}^{\prime}(t)=\frac{a u_{6}(t)\left(b_{h} u_{1}(t)+\widetilde{b}_{h} u_{3}(t)\right)}{H_{0}}-\gamma_{h} u_{2}(t)-\delta_{h} u_{2}(t) \\
& u_{3}^{\prime}(t)=\gamma_{h} u_{2}(t)-\frac{a \widetilde{b}_{h} u_{3}(t) u_{6}(t)}{H_{0}}-w_{3}(t) u_{3}(t) \\
& u_{4}^{\prime}(t)=\delta_{h} u_{2}(t) \\
& u_{5}^{\prime}(t)=-\frac{a b_{m} u_{2}(t) u_{5}(t)}{H_{0}}+g(M(t))-\mu_{m} u_{5}(t)-w_{m}(t) u_{5}(t) \\
& u_{6}^{\prime}(t)=\frac{a b_{m} u_{2}(t) u_{5}(t)}{H_{0}}-\mu_{m} u_{6}(t)-w_{m}(t) u_{6}(t)
\end{aligned}
$$

for $t \in[0, T]$, with $0 \leq w_{1}, w_{3} \leq w_{H}, 0 \leq w_{m} \leq w_{M}$ and $w=\left(w_{1}, w_{3}, w_{m}\right)$. The variables $A_{j}$ are the positive weights associated with the control variables $w_{j}, j=1,3, m$, respectively.

Lemma 4.1. There exists an optimal control $w^{\star}=\left(w_{1}^{\star}(t), w_{3}^{\star}(t), w_{m}^{\star}(t)\right)$ such that

$$
\mathcal{J}\left(w_{1}^{\star}, w_{3}^{\star}, w_{m}^{\star}\right)=\min _{w \in W} \mathcal{J}\left(w_{1}, w_{3}, w_{m}\right)
$$

under the constraint $\left(u_{1}, u_{2}, u_{3}, u_{4}, u_{5}, u_{6}\right)$ is a solution of (11).

Proof. Since all control inputs $w_{1}(t), w_{3}(t)$ and $w_{m}(t)$ are piecewise continuous functions that takes its values in a positively bounded set $W$ and $u_{2}(t)$ is continuous, the corresponding set of controls and the state variables is nonempty, closed and convex on $W$. The objective function is convex and coercive.

Pontryagin's maximum principle is used to find the best possible control for taking a dynamical system from one state to another. It states that it is necessary for any optimal control along with the optimal state trajectory to solve the so-called Hamiltonian system [10]. We state the lemma below.

Lemma 4.2. There exist the adjoint variables $\lambda_{i}, i=1,2, \cdots, 6$ of the system (11) that satisfy the following backward in time system of ordinary differential equations:

$$
\begin{aligned}
& -\frac{d \lambda_{1}}{d t}=\lambda_{1}\left(\frac{-a b_{h} u_{6}}{H_{0}}-w_{1}\right)+\lambda_{2} \frac{a b_{h} u_{6}}{H_{0}} \\
& -\frac{d \lambda_{2}}{d t}=1+\lambda_{2}\left(-\gamma_{h}-\delta_{h}\right)+\lambda_{3} \gamma_{h}+\lambda_{4} \delta_{h}-\lambda_{5} \frac{a b_{m} u_{5}}{H_{0}}+\lambda_{6} \frac{a b_{m} u_{5}}{H_{0}} \\
& -\frac{d \lambda_{3}}{d t}=\lambda_{2} \frac{a \widetilde{b}_{h} u_{6}}{H_{0}}+\lambda_{3}\left(\frac{-a \widetilde{b}_{h} u_{6}}{H_{0}}-w_{3}\right) \\
& -\frac{d \lambda_{4}}{d t}=0 \\
& -\frac{d \lambda_{5}}{d t}=\lambda_{5}\left(\frac{-a b_{m} u_{2}}{H_{0}}+\frac{\partial g}{\partial u_{5}}\right)-\lambda_{5}\left(\mu_{m}+w_{m}\right)+\lambda_{6} \frac{a b_{m} u_{2}}{H_{0}} \\
& -\frac{d \lambda_{6}}{d t}=-\lambda_{1} \frac{a b_{h} u_{1}}{H_{0}}+\lambda_{2} \frac{a b_{h} u_{1}+a \widetilde{b}_{h} u_{3}}{H_{0}}-\lambda_{3} \frac{a \widetilde{b}_{h} u_{3}}{H_{0}}+\lambda_{5} \frac{\partial g}{\partial u_{6}}-\lambda_{6}\left(\mu_{m}+w_{m}\right)
\end{aligned}
$$

with the transversality condition $\lambda(T)=0$. 
Proof. Using the Hamiltonian for (11), we have

$$
\begin{aligned}
\mathcal{H}= & \mathcal{L}\left(w_{1}, w_{3}, w_{m}\right)+\lambda_{1}(t) u_{1}^{\prime}(t)+\lambda_{2}(t) u_{2}^{\prime}(t)+\lambda_{3}(t) u_{3}^{\prime}(t)+\lambda_{4}(t) u_{4}^{\prime}(t)+\lambda_{5}(t) u_{5}^{\prime}(t)+\lambda_{6}(t) u_{6}^{\prime}(t) \\
= & \frac{1}{2}\left(u_{2}^{2}+A_{1} w_{1}^{2}+A_{3} w_{3}^{2}+A_{m} w_{m}^{2}\right) \\
& +\lambda_{1}\left(-\frac{a b_{h} u_{6} u_{1}}{H_{0}}-w_{1} u_{1}\right)+\lambda_{3}\left(\gamma_{h} u_{2}-\frac{a \widetilde{b}_{h} u_{3} u_{6}}{H_{0}}-w_{3} u_{3}\right) \\
& +\lambda_{2}\left(\frac{a u_{6}\left(b_{h} u_{1}+\widetilde{b}_{h} u_{3}\right)}{H_{0}}-\gamma_{h} u_{2}-\delta_{h} u_{2}\right)+\lambda_{4}\left(\delta_{h} u_{2}\right) \\
& +\lambda_{5}\left(-\frac{a b_{m} u_{2} u_{5}}{H_{0}}+g(M)-\mu_{m} u_{5}-w_{m} u_{5}\right) \\
& +\lambda_{6}\left(\frac{a b_{m} u_{2} u_{5}}{H_{0}}-\mu_{m} u_{6}-w_{m} u_{6}\right) .
\end{aligned}
$$

Therefore, finding the partial derivatives of $\mathcal{H}$ with respect to $u_{i}$ 's, $i=1,2, \cdots, 6$, we have

$$
\begin{aligned}
& \frac{\partial \mathcal{H}}{\partial u_{1}}=\lambda_{1}\left(\frac{-a b_{h} u_{6}}{H_{0}}-w_{1}\right)+\lambda_{2} \frac{a b_{h} u_{6}}{H_{0}} \\
& \frac{\partial \mathcal{H}}{\partial u_{2}}=1+\lambda_{2}\left(-\gamma_{h}-\delta_{h}\right)+\lambda_{3} \gamma_{h}+\lambda_{4} \delta_{h}-\lambda_{5} \frac{a b_{m} u_{5}}{H_{0}}+\lambda_{6} \frac{a b_{m} u_{5}}{H_{0}} \\
& \frac{\partial \mathcal{H}}{\partial u_{3}}=\lambda_{2} \frac{a \widetilde{b}_{h} u_{6}}{H_{0}}+\lambda_{3}\left(\frac{-a \widetilde{b}_{h} u_{6}}{H_{0}}-w_{3}\right) \\
& \frac{\partial \mathcal{H}}{\partial u_{4}}=0 \\
& \frac{\partial \mathcal{H}}{\partial u_{5}}=\lambda_{5}\left(\frac{-a b_{m} u_{2}}{H_{0}}+\frac{\partial g}{\partial u_{5}}\right)-\lambda_{5}\left(\mu_{m}+w_{m}\right)+\lambda_{6} \frac{a b_{m} u_{2}}{H_{0}} \\
& \frac{\partial \mathcal{H}}{\partial u_{6}}=-\lambda_{1} \frac{a b_{h} u_{1}}{H_{0}}+\lambda_{2} \frac{a b_{h} u_{1}+a \widetilde{b}_{h} u_{3}}{H_{0}}-\lambda_{3} \frac{a \widetilde{b}_{h} u_{3}}{H_{0}}+\lambda_{5} \frac{\partial g}{\partial u_{6}}-\lambda_{6}\left(\mu_{m}+w_{m}\right) .
\end{aligned}
$$

Then the adjoint system is defined by $\frac{d \lambda_{i}}{d t}=-\frac{\partial \mathcal{H}}{\partial u_{i}}$ for $i=1,2, \cdots, 6$.

Theorem 4.3. The optimal control variables are given by

$$
\begin{aligned}
w_{1}^{\star}(t) & =\max \left(0, \min \left(\frac{\lambda_{1} u_{1}}{A_{1}}, w_{H}\right)\right) \\
w_{3}^{\star}(t) & =\max \left(0, \min \left(\frac{\lambda_{3} u_{3}}{A_{3}}, w_{H}\right)\right) \\
w_{m}^{\star}(t) & =\max \left(0, \min \left(\frac{\lambda_{5} u_{5}+\lambda_{6} u_{6}}{A_{m}}, w_{M}\right)\right)
\end{aligned}
$$

Proof. By the Pontryagin maximum principle, the optimal control $w^{\star}$ minimizes, at each instant $t$, the Hamiltonian given by (12). We have

$$
\frac{\partial H}{\partial w_{j}}=0, \quad \text { for all } \quad j=1,3, m \text { at } \quad w_{j}=w_{j}^{\star} .
$$

Therefore, we get

$$
\frac{\partial \mathcal{H}}{\partial w_{1}}=A_{1} w_{1}-\lambda_{1} u_{1}, \quad \frac{\partial \mathcal{H}}{\partial w_{3}}=A_{3} w_{3}-\lambda_{3} u_{3}, \quad \frac{\partial \mathcal{H}}{\partial w_{m}}=A_{m} w_{m}-\lambda_{5} u_{5}-\lambda_{6} u_{6},
$$

and

$$
w_{1}=\frac{\lambda_{1} u_{1}}{A_{1}}, \quad w_{3}=\frac{\lambda_{3} u_{3}}{A_{3}}, w_{m}=\frac{\lambda_{5} u_{5}+\lambda_{6} u_{6}}{A_{m}} .
$$




\subsection{Numerical Simulation of Optimal Control}

Numerical simulations show the difference in minimizing the infected human during the dengue outbreak between the three methods: vaccination, vector control, and the combination of the vaccination and vector control.

Table 1: Parameter values used in the numerical simulations.

\begin{tabular}{c|c|c}
\hline Symbol & Description & Values \\
\hline \hline$a$ & number of human beaten per mosquito & 1 \\
$b_{h}$ & probability of becoming infected & 0.75 \\
$\widetilde{b}_{h}$ & probability of becoming infected again & 0.375 \\
$\alpha_{h}$ & growth rate of human & 0.0045 \\
$\gamma_{h}$ & recovery rate of human from first infection & 0.328833 \\
$\delta_{h}$ & recovery rate of human from the second infection & 0.1666 \\
$b_{m}$ & probability of becoming infectious & 0.375 \\
$\mu_{m}$ & death rate of mosquito & 0.02941 \\
$\alpha_{m}$ & growth rate of mosquito & 0.025 \\
\hline
\end{tabular}

The parameters are presented in Table 1, which is taken from [3]. Notice that $\alpha_{m}<\mu_{m}$, by Theorem 2.4 the global stability corresponds to $E_{1}$. Here $E_{2}$ is biologically not meaningful in this situation. The author estimated some from Indonesia, where environmental conditions are similar to the Philippines. The control weights $A_{1}$ and $A_{3}$ are the efforts in vaccinating the human population. In contrast, the control weights $A_{m}$ is the effort to eliminate the mosquito population by means of administering insecticides. Since primary susceptible humans are readily available in the population compared to the secondary susceptible humans, the efforts used in vaccinating them would be less than the effort exerted in vaccinating the secondary susceptible. Thus, $A_{3}$ is set higher than $A_{1}$. While insecticide administration in susceptible mosquitoes and infected mosquitoes uses the same effort and achieves a similar result. Hence, we initially set the control weights as $A_{1}=0.1, A_{3}=1$ and $A_{m}=1$. Note that the values of $A_{1}, A_{3}, A_{m}$ do not change the convergence of optimal control.

The optimality of the system is numerically solved using Algorithm 1 with $\epsilon=0.01$.

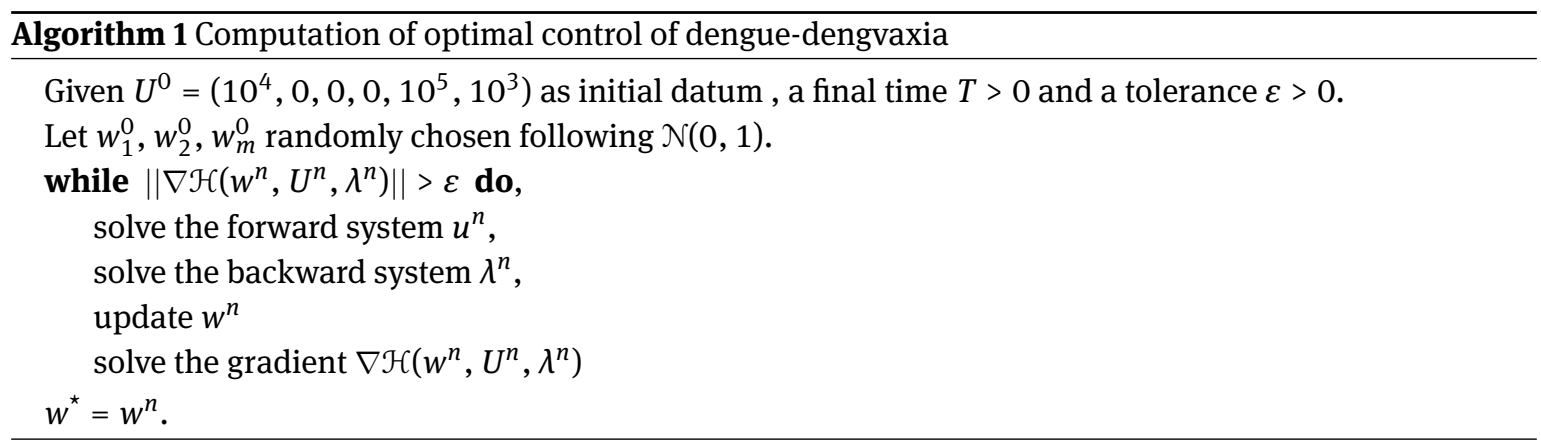

Figure 2 shows in minimizing the infected human that the combination of Dengvaxia and vector control is the most effective method. It would only take 30 days to reach equilibrium, resulting in the total elimination of infected humans with a maximum of $12.55 \%(1,255)$ infected humans over time. Nevertheless, vector control stands out if we compare only the vaccination and vector control method. It would only take 34 days with a maximum population of $19.68 \%(1,968)$ infected humans for vector control to eliminate infected humans. 


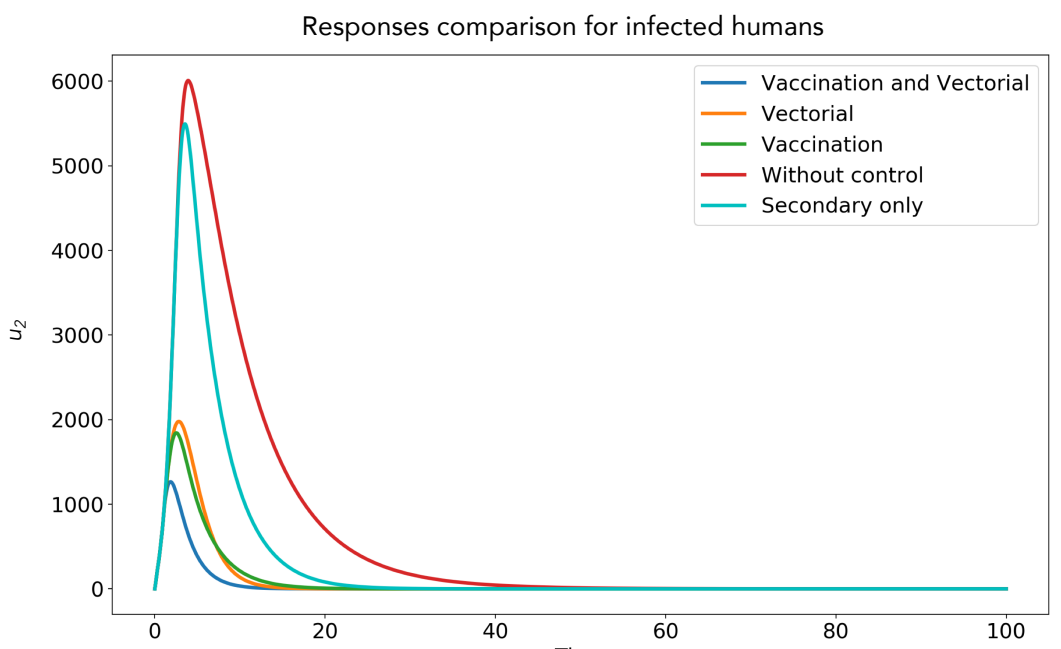

Figure 2: Behaviour of infected humans $I_{h}$ with respect to time without control (red), for the optimal control related to the vaccination only (green), related to the vector only (orange), and with both control (blue). Cyan curve corresponds to optimal control of vaccination of secondary humans only.

In comparison, vaccination takes 45 days, with $18.42 \%(1,842)$ infected humans. Without control, infected humans would slowly decrease after reaching $60.07 \%(6,007)$ but would never annihilate. Vaccinating secondary humans would only take 48 days to reach equilibrium with a maximum of $54.94 \%(5,494)$ infected humans over time.
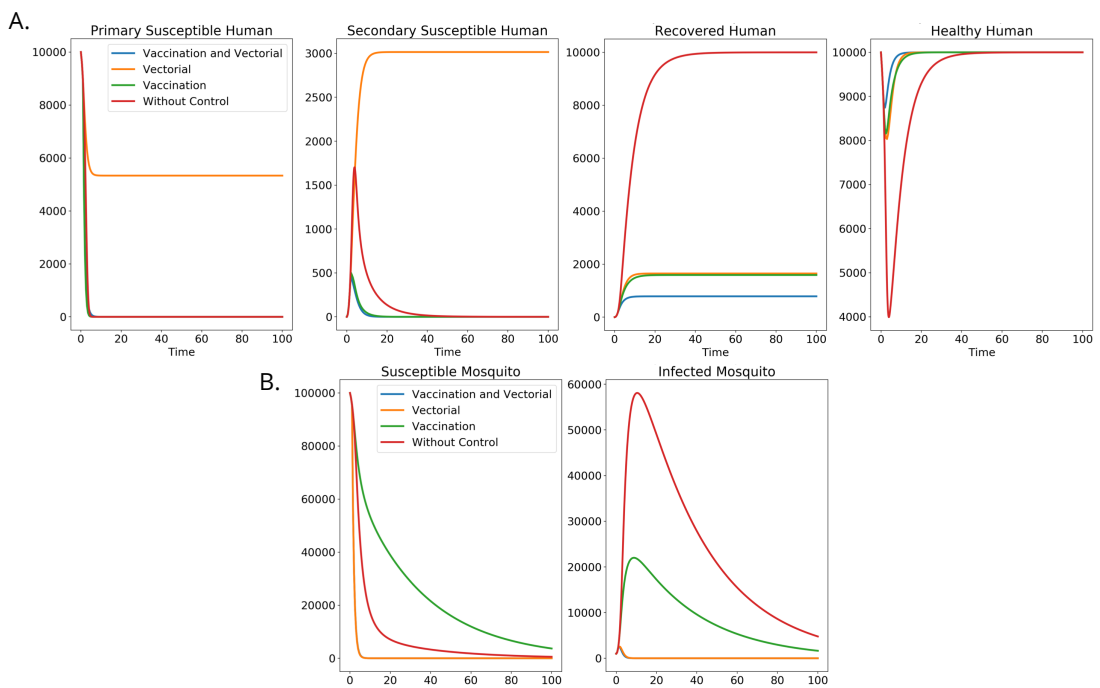

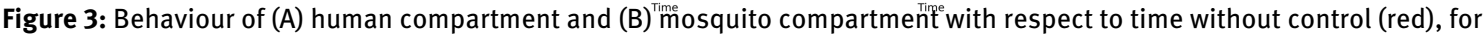
the optimal control related to the vaccination only (green), related to the vector only (orange), and with both control (blue).

Figure 3 shows no significant difference between the three methods concerning the primary and secondary susceptibles to reach its equilibrium point. It takes seven and a half days for vaccination and 14 days to combine vaccination and vector control to reach zero primary susceptible individuals. Moreover, it takes 42 days for vaccination and 30 days for the combination to reach zero secondary susceptible individuals. For vector control, it takes 20 days to reach an equilibrium of 53.36\% (5336) primary susceptible humans and 34 days to reach $30.15 \%$ (3015) secondary susceptible humans.

Note that in the human compartment, the total immunity to dengue by means of implementing the Dengvaxia vaccine is denoted by $T_{h}$ which is given by $T_{h}(t)=w_{1}(t) u_{1}(t)+w_{3}(t) u_{3}(t)$. Thus, a healthy human com- 
bines immune human $T_{h}$, primary susceptible $u_{1}$, secondary susceptible $u_{3}$, and the recovered $u_{4}$ humans. The figure shows that the combination of vaccination and vector control is the best method in maximizing the healthy human population. It only takes 26 days to combine vaccination and vector control methods to reach the equilibrium of healthy humans. Its minimum population is $8.73 \%(8,734)$ on day 1.8 .

In contrast, there is no significant difference in the vector and vaccination method alone in the healthy human population. The vector method takes 29 days to reach its equilibrium with $8.02 \%(8,021)$ minimum population on 2.8 days. The vaccination required 39 days to reach an equilibrium with $8.16 \%(8,157)$ minimum population on 2.5 days. Without any control strategies applied to the healthy human population, it requires a much higher time to reach its equilibrium with 4\% $(4,000)$ minimum population.

For the recovered human compartment, the figure shows that the human population would eventually recover through time without control strategies applied to the variables. It supports that dengue infection lasts only three to seven days following the infectious mosquito bite, and a spontaneous, full health recovery follows. However, comparing the three control methods, the combination of vaccination and vector control methods stands out. It only requires 26 days to reach its equilibrium at $0.79 \%$ (787) recovered human population. At the same time, there is no significant difference between vaccination alone and vector control alone. Both require 32 days to reach its equilibrium at $1.59 \%(1,590)$ and $1.65 \%(1,646)$ recovered human, respectively.

Now, minimizing the susceptible mosquito population, no control applied to the variables is better than vaccination. It decreases faster with $0.56 \%$ (556) minimum susceptible mosquito population while vaccination decreases slower with 3.69\% $(3,692)$ minimum population at the end of time. Nevertheless, the vector control method and the combination of vaccination and vector control are the better methods for controlling the mosquito population. They annihilate the susceptible mosquito population.

Minimizing the infected mosquito, either vector control alone or combining vaccination and vector control is the best method. There is no significant difference between the two. They both require minimum time for the infected mosquito to reach zero population and with only $2.6 \%$ (2596) and 2.52\% (2521) maximum population for the vector control only and the combination, respectively. However, vaccination is better compared to the one without control. The infected mosquito has a $58.09 \%(58,092)$ maximum population without applying a control strategy, while the vaccination has a $22 \%(22,006)$ maximum population.

Now, let us show the controlled variable's behavior by comparing the vaccination, vector control only, and the combination of vaccination and vector control.
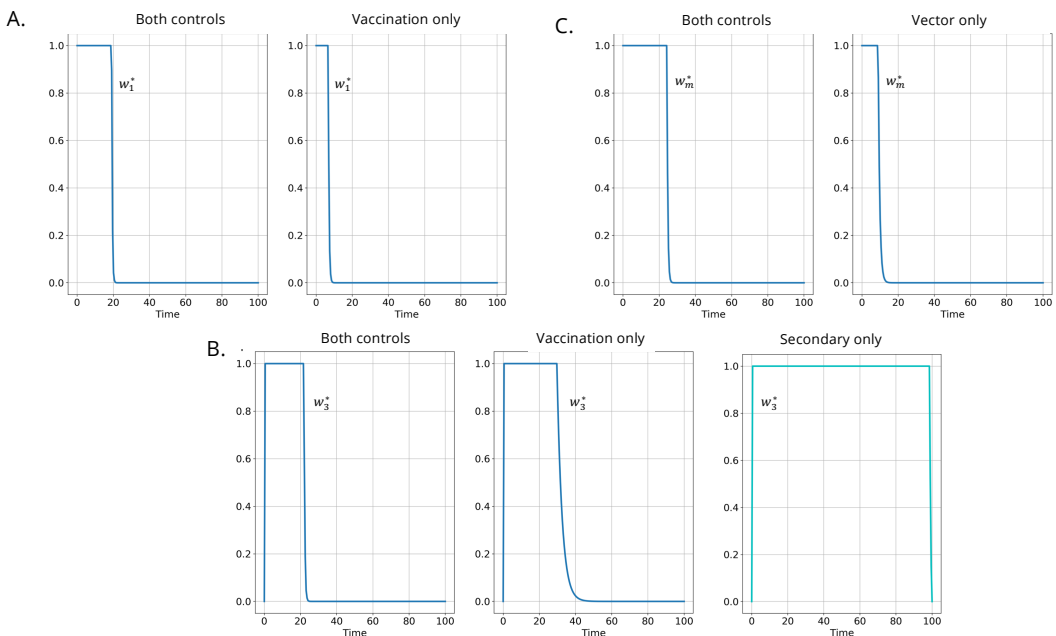

Figure 4: Optimal control of the (A) primary susceptible and (B) secondary susceptible human compartment using vaccination only versus the combination of both control strategies. Optimal control of (C) mosquitoes compartment using vector control only versus the combination of both control strategies. Cyan curve represents optimal control of vaccination of secondary humans only 
Figure 4 shows that concerning vaccination only, vaccinating the primary susceptible human population requires a shorter time compared to the secondary susceptible human. It takes ten days and 40 days to vaccinate primary and secondary susceptible human populations, respectively. Nevertheless, applying both control strategies takes only approximately 20-22 days. While minimizing the mosquito population, the vector control method is better than combining the two strategies. It takes approximately 15-18 days to administer insecticide to the mosquito population while applying both control strategies takes 22-24 days.

\section{Conclusion}

Following Ross' model, we build a mathematical model for dengue where secondary susceptible individuals partake in the infection. As proposed by Sanofi, the dengue vaccine is recommended to individuals who have been infected by one serotype of dengue [16]. In our study, we have shown that vaccinating secondary susceptible humans only is not ideal. It requires constant effort and takes a long time to vaccinate them, as observed in Figure 2 (cyan curve) and Figure 4. Instead, it is better to vaccinate the primary susceptible humans. However, since safe vaccines for primary susceptible humans do not exists to date, the application of vector control to minimize infected humans is a better counter-strategy.

Our results show that vector control is better than vaccination to maximize the healthy human population in a short period. Thus, using cyclopoid copepods (Mesocyclops spp.) or the releasing of mosquitoes modified to carry a Wolbachia bacteria is better than vaccination. However, further study with good design, data quality, and appropriate statistics is needed for this.

Financial Support: This research received no specific grant from any funding agency, commercial or nonprofit sectors.

Conflict of Interests Statement: The author has no conflicts of interest to disclose.

Ethics Statement: This research did not require ethical approval.

Acknowledgement: The author thanks the anonymous referees for their comments and invaluable remarks and suggestions.

The author expresses her immeasurable appreciation and deepest gratitude to her adviser Prof. Youcef Mammeri for the invaluable support for numerous discussions, inspiring remarks, and valuable suggestions that influenced this paper's final form.

\section{References}

[1] M. Aguiar and N. Stollenwerk. Mathematical models of dengue fever epidemiology: multi-strain dynamics, immunological aspects associated to disease severity and vaccines. Communication in Biomathematical, 1, 2017. URL https://doi.org/10. 5614/cbms.2017.1.1.1.

[2] M. Aguiar, N. Stollenwerk, and S. Halstead. The impact of the newly licensed dengue vaccine in endemic countries. PLoS Neglected Tropical Diseases, 10, 2016. URL https://doi.org/10.1371/journal.pntd.0005179.

[3] I. Bakach and J. Braselton. A survey of mathematical models of dengue fever. Journal of Computer Science \& Systems Biology, 08, 01 2015. URL https://doi.org/10.4172/jcsb.1000198.

[4] S. Bhatt, P. Gething, O. Brady, J. Messina, A. Farlow, C. Moyes, J. Drake, J. Brownstein, A. Hoen, O. Sankoh, M. Myers, D. George, T. Jaenisch, W. Wint, C. Simmons, T. Scott, J. Farrar, and S. Hay. The global distribution and burden of dengue. Nature, 496:504-507, 04 2013. URL https://doi.org/10.1038/nature12060.

[5] P.-A. Bliman, D. Cardona-Salgado, Y. Dumont, and O. Vasilieva. Implementation of control strategies for sterile insect techniques. Mathematical Biosciences, 314:43-60, 2019. URL https://doi.org/10.1016/j.mbs.2019.06.002.

[6] A. S. Carvalho, S. Silva, and I. Charret. Mathematical modeling of dengue epidemic: Control methods and vaccination strategies. Theory in Biosciences, 138, 2015. URL https://doi.org/10.1007/s12064-019-00273-7. 
[7] M. Derouich, A. Boutayeb, and E. Twizell. A model of dengue fever. Biomedical Engineering Online, 2, 2003. URL https: //doi.org/10.1186/1475-925X-2-4.

[8] Report 8: Monthly dengue report: January 1 - August 31, 2019 (MW 1-35). DOH Department of Health, Accessed April 22, 2020 2019. URL https://www.doh.gov.ph/sites/default/files/statistics/2019\%20Dengue\%20Monthly\%20Report\%20No.\%208. pdf.

[9] E. Iboi and A. Gumel. Mathematical assessment of the role of dengvaxia vaccine on the transmission dynamics of dengue serotypes. Mathematical Biosciences, 304, 2018. URL https://doi.org/10.1016/j.mbs.2018.07.003.

[10] S. Lenhart and J. Workman. Optimal Control Applied to Biological Models. 012007.

[11] N. Nuraini, E. Soewono, and K. Sidarto. Mathematical model of dengue disease transmission with severe dhf compartment. Bull. Malays. Math. Sci. Soc, 30:143-157, 2007. URL http://emis.dsd.sztaki.hu/journals/BMMSS/pdf/v30n2/v30n2p7.pdf.

[12] Senate invites Aquino to vaccine probe. The Philippine Star, Accessed April 7, 2020 2017. URL https://www.philstar.com/ headlines/2017/12/12/1767890/senate-invites-aquino-vaccine-probe.

[13] P. Pongsumpun, I.-M. Tang, and N. Wongvanich. Optimal control of the dengue dynamical transmission with vertical transmission. Advances in Difference Equations, 2019. URL https://doi.org/10.1186/s13662-019-2120-6.

[14] S. Side and M. Noorani. Seir model for transmission of dengue fever. International Journal on Advance Science, Engineering \& Information Technology, 2(5), 2012. URL https://doi.org/10.18517/ijaseit.2.5.217.

[15] P. Van den Driessche and J. Watmough. Reproduction numbers and sub-threshold endemic equilibria for compartmental models of disease transmission. Mathematical Biosciences, 180(1-2):29-48, 2000. URL https://doi.org/10.1016/S00255564(02)00108-6.

[16] Dengue vaccine: WHO position paper. World Health Organization, weekly epidemiological record edition, September 2018. URL https://apps.who.int/iris/handle/10665/274316.

[17] H. Yang and C. Ferreira. Assessing the effects of vector control on dengue transmission. Applied Mathematics and Computation, 198:401-413, 2008. URL https://doi.org/10.1016/j.amc.2007.08.046.

[18] L. Yauch and S. Shresta. Dengue virus vaccine development. Advances in Virus Reserch, pages 315-72, 2014. URL https: //doi.org/10.1016/B978-0-12-800098-4.00007-6. 